Ann. Biol. anim. Bioch. Biophys., I965, 5 (2), 309-315.

\title{
SYNDROME MYOPATHIE-DYSPNÉE : INFLUENCE DE LA PROPHYLAXIE PAR LE SÉLENIUM SUR LA COMPOSITION BIOCHIMIQUE DU PLASMA CHEZ LE VEAU
}

\author{
M. LAMAND \\ Arec la collaboration technique de P. Ciragiacd, Nicole Berthelot, \\ J. Bellanger et de J. Overwater \\ Laboratoire de Nutrition minérale, \\ Institut national de la Recherche agronomique, \\ École nationale vétérinaire, Alfort (Seine)
}

SOMMAIRE

Le taux de transaminase glutamique oxalacétique plasmatique de 25 veaux engraissés à la mamelle, dont les mères avaient, selon les cas, reçu ou non du sélénium, a été mesuré.

Il n'a pas été observé de différence significative entre les groupes des veaux malades et sains sans prophylaxie au sélénium; en revanche le groupe traité par le sélénium présente un taux de transaminase glutamique oxalacétique significativement inférieur à celui des veaux non soumis à la prophylaxie.

Le taux de fer plasmatique est significativement plus élevé chez les malades.

\section{INTRODUC'TION}

Les animaux habituellement atteints de myopathie sont essentiellement des veaux engraissés à la mamelle sans aucune autre nourriture, de race Limousine ou Charolaise et dont la coloration claire des muscles est particulièrement recherchée.

En I958 Mưru et al., ont montré que l'administration de sélénium chez l'agneau constituait une prophylaxie efficace contre cette affection. En I959, SHARman, BLAXTER et WrLson aboutissent aux mêmes conclusions chez le veau. Nous avons suivi les effets de l'administration de sélénium par le dosage du taux de transami- 
nase glutamique oxalacétique (TGO) du plasma. L'élévation de ce taux est un excellent moyen de diagnostic de l'atteinte de la cellule musculaire. La cellule musculaire ne libère cette enzyme dans le plasma qu'en cas de lésion (KU'TLLER et MARBLE, I958).

D'autre part chez les animaux nourris exclusivement au lait il nous a paru intéressant de suivre les taux de fer et de cuivre. De plus, comme chez les malades les symptômes peuvent parfois faire penser à une hypomagnésiémie d'autant plus vraisemblable que ces animaux ont un régime naturellement pauvre en magnésium, nous avons également étudié les variations des taux de magnésium dans le plasma.

\section{MATÉRIEL, ET MÉTHODES}

$$
\mathbf{I}^{\mathrm{o}} \text { Animaux }
$$

Cette étude a été menée sur un total de 25 veaux provenant de 15 exploitations. Huit de ces I 5 exploitations ne pratiquaient pas de prophylaxie particulière et 7 pratiquaient une prophylaxie par le sélénium de la façon suivante :

Les mères recevaient un complément minéral classique renfermant $\mathrm{I} 7,6 \mathrm{~g}$ de sélénite de sodium par $100 \mathrm{~kg}$, distribué à raison de I $50 \mathrm{~g}$ par vache et par jour. En effet, Mc ConNel (1963) a montré que le sélénium était transmis au jeune par le lait.

Les prélèvements de sang ont été effectués:

a) chez des animaux présentant des signes cliniques de myopathie dans les fermes sans prophylaxie ;

b) chez des animaux cliniquement sains, considérés comme témoins, des mêmes fermes;

c) chez des animaux dans des exploitation pratiquant la prophylaxie par le sélénium (tous cliniquement sains).

\section{$2^{\circ}$ Modalités de prélèvement}

Le sang a été prélevé dans des tubes contenant de l'héparine. Certains tubes ont été expédiés frais dans les meilleurs délais, les autres ont été congelés immédiatement et transportés à $-9^{\circ} \mathrm{C}$. Nous avons vérifié qu'il n'y avait pas de différence sensible dans les résultats des dosages entre ces deux modalités de prélèvement, la TGO résistant aussi bien à la température ordinaire pendant quelques jours qu'à une congélation prolongée.

\section{$3^{\mathbf{0}}$ Dosage de la transaminase glutamique oxalacétique}

La technique utilisée est celle de Rerrman et Frankel (I957). Cette méthode nous a foumi des chiffres un peu plus élevés que ceux cités par certains auteurs (BLINCOE et DyE, I958; KUTTLER et MARBLE, I958) qui employaient la méthode de CABALD, LeEPER et Wroblewski (I956). En effet, la réaction enzymatique sur le substrat dans la méthode que nous avons employée se fait à $37^{\circ} \mathrm{C}$ en bain-marie au lieu de la température du laboratoire. Ceci explique les chiffres plus élevés, mais ce procédé permet une meilleure standardisation de la méthode.

Une atteinte hépatique élève également dans de moindres proportions le taux de TGO plasmatique mais le plus généralement il n'y a pas de lésion hépatique dans le tableau clinique du syndrome Myopathie-Dyspnée.

\section{$4^{\circ}$ Dosage des minéraux plasmaliques}

Le magnésium a été dosé par la méthode au jaune de titane modifiée par LEwIs (Ig60).

Le calcium a été dosé par photométrie de flamme (appareil Eppendorf).

Le dosage colorimétrique du fer a été fait par la méthode à l'orthophénanthroline (méthode de Henry, Sobel et ChIAMori, I958).

Le dosage colorimétrique du cuivre a été fait par la méthode à la cuproïne (Jérone et SсHмiтt, 1954). 


\section{$5^{\circ}$ Interprétation statistique}

Les données de TGO et de fer ont subi une transformation logarithmique de façon à rendre normale la distribution de ces variables permettant l'exploitation statistique.

Les résultats (moyenne et intervalle de confiance) ont été calculés à partir des données transformées, mais exprimés en unités réelles, ce qui donne un intervalle de confiance dissymétrique par rapport à la moyenne.

\section{RÉSULTATS}

Le tableau I indique les valeurs respectives de la transminase en fonction de l'état clinique et de la prophylaxie mise en œuvre.

Aucun malade n'est apparu pendant un an parmi les animaux dont les mères avaient reçu du sélénium.

\section{TABIEAU I}

Taux de transaminase glutamique oxalacétique du plasma en fonction de l'état clinique

\begin{tabular}{|c|c|c|c|c|c|}
\hline \multicolumn{2}{|c|}{ Animaux malades } & \multicolumn{2}{|c|}{ Animaux sains sans prophylaxie } & \multicolumn{2}{|c|}{ Animaux sains avec prophylaxie } \\
\hline $\begin{array}{l}\text { Numéro de } \\
\text { l'animal }\end{array}$ & Unités de TGO $\left({ }^{1}\right)$ & $\begin{array}{l}\text { Numéro de } \\
\text { l'animal }\end{array}$ & Unités de TGO (1) & $\begin{array}{l}\text { Numéro de } \\
\text { l'animal }\end{array}$ & Unités de TGO(1) \\
\hline $\begin{array}{r}1 \\
2 \\
4 \\
6 \\
8 \\
9 \\
17 \\
19 \\
21\end{array}$ & $\begin{array}{l}407 \\
367 \\
365 \\
268 \\
161 \\
177 \\
400 \\
470 \\
442 \\
\end{array}$ & $\begin{array}{r}3 \\
5 \\
7 \\
18 \\
20 \\
22\end{array}$ & $\begin{array}{l}242 \\
300 \\
137 \\
139 \\
210 \\
356\end{array}$ & $\begin{array}{l}10 \\
11 \\
12 \\
13 \\
14 \\
15 \\
16 \\
23 \\
25 \\
26\end{array}$ & $\begin{array}{r}65 \\
93 \\
112 \\
65 \\
106 \\
104 \\
89 \\
202 \\
125 \\
164\end{array}$ \\
\hline \multicolumn{2}{|c|}{ Moyenne $\left({ }^{2}\right): 318$} & \multicolumn{2}{|c|}{ Moyenne $\left({ }^{2}\right): 216$} & \multicolumn{2}{|c|}{ Moyenne $\left({ }^{2}\right): 106$} \\
\hline \multicolumn{2}{|c|}{$\begin{array}{l}\text { Intervalle de confiance } 95 \% \text { de la } \\
\text { moyenne allant de } 236 \text { a } 429 \text {. } \\
\text { Intervalle de con fiance } 95 \% \text { de la } \\
\text { population } 130 \text { à } 782 \text {. }\end{array}$} & \multicolumn{2}{|c|}{$\begin{array}{c}\text { Intervalle de confiance } 95 \% \text { de la } \\
\text { moyenne allant de } 143 \dot{a} 326 .\end{array}$} & \multicolumn{2}{|c|}{$\begin{array}{l}\text { Intervalle de confiance } 95 \% \text { de la } \\
\text { moyenne allant de } 82 \text { à } 138 \text {. } \\
\text { Intervalle de confiance } 95 \% \text { de la } \\
\text { population } 47 \text { à } 240 .\end{array}$} \\
\hline
\end{tabular}

(1) Une unité de TGO (Unité ReItuann FrankeL) est définie comme lal coloration produite dans les conditions du dosage par $1 \mu \mathrm{g}$ d'acide pyruvique/ml de plasma.

(2) Après transformation logarithmique permettant d'obtenir pour ces données une répartition normale et retour à l'unité initiale.

Le groupe des animaux sains avec prophylaxie montre une différence hautement significative avec les 2 groupes d'animaux sans prophylaxie (test $F=20,4$, limite I p. IOO $=5,72$ ).

La différence entre les animaux sains sans prophylaxie et malades n'est, par contre, pas significative. 
TABLEAU 2

Teneur en fer plasmatique $(\mu \mathrm{g} ! 100 \mathrm{ml})$. Classification en 3 groupes d'après le taux de transaminase

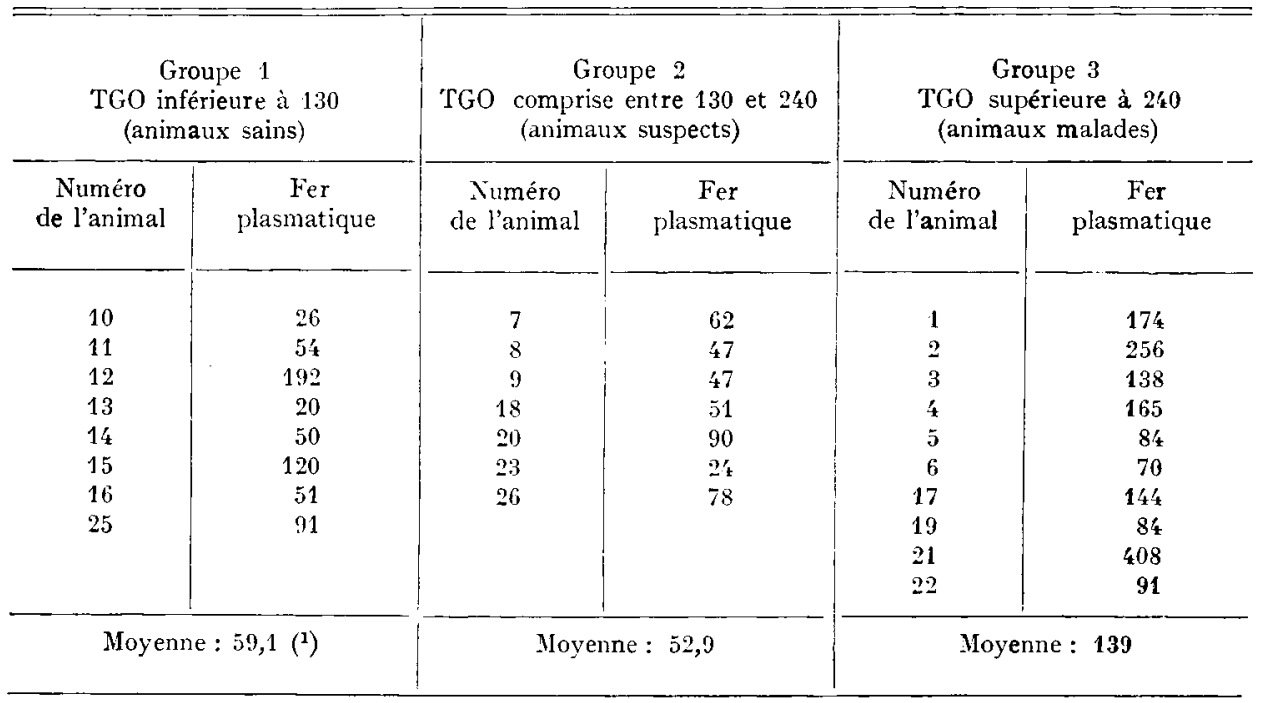

(i) Après transformation logarithmique permettant d'obtenir pour ces données une répartition normale et retour à l'unité initiale.

TABLEAU 3

Taux de minéraux plasmatiques des animaux malades et des témoins

\begin{tabular}{|c|c|c|c|c|c|c|c|c|c|}
\hline \multirow{2}{*}{$\begin{array}{l}\text { Numéro } \\
\text { de } \\
\text { lanimal }\end{array}$} & \multicolumn{4}{|c|}{ Veaux cliniquement malades } & \multirow{2}{*}{$\begin{array}{c}\text { Numéro } \\
\text { de } \\
\text { l'animal }\end{array}$} & \multicolumn{4}{|c|}{ Veaux cliniquement sains } \\
\hline & $\left\{\begin{array}{c}\text { Magnésium } \\
(\mathrm{mg} / 100 \\
\mathrm{ml})\end{array}\right.$ & $\begin{array}{c}\text { Calcium } \\
(\mathrm{mg} / 100 \\
\mathrm{nl})\end{array}$ & $\left\{\begin{array}{c}\text { Fer } \\
(\gamma / 100 \\
\mathrm{ml})\end{array}\right.$ & $\left|\begin{array}{c}\text { Cuivre } \\
(\gamma / 100 \\
\mathrm{ml})\end{array}\right|$ & & $\left\{\begin{array}{l}\text { Magnésium } \\
(\mathrm{mg} / 100 \\
\mathrm{ml})\end{array}\right.$ & $\left\{\begin{array}{c}\text { Calcium } \\
(\mathrm{mg} / 100 \\
\mathrm{ml})\end{array}\right.$ & $\left(\begin{array}{c}\text { Fer } \\
(\gamma / 100 \\
\mathrm{ml}\end{array}\right)$ & $\begin{array}{r}\text { Cuivre } \\
(y / 100 \\
\mathrm{ml})\end{array}$ \\
\hline 1 & 1,24 & 11,9 & 174 & & 3 & 1,98 & $12, \overline{5}$ & 138 & \\
\hline 2 & 2,26 & 12,1 & 256 & & ร & 2,22 & 12,4 & 84 & \\
\hline 4 & 2,53 & 10,1 & 185 & & 7 & 1,74 & 11,4 & 62 & \\
\hline 6 & 2,98 & 12,1 & 70 & & 10 & 1,96 & 12,7 & 26 & 100 \\
\hline 8 & 1,72 & 12,6 & 47 & & 11 & 1,72 & 12,8 & $5 ! 4$ & 30 \\
\hline 9 & 1,98 & 12.6 & 47 & 174 & 12 & 2,03 & 12,5 & 192 & 81 \\
\hline 17 & 2,55 & 17,0 & 144 & 114 & 13 & 1,70 & 12,4 & 20 & 86 \\
\hline 19 & 2,01 & 12,3 & 84 & 135 & 14 & 1,93 & 11,4 & 50 & 105 \\
\hline 21 & 2,10 & 12,3 & 408 & 102 & 15 & 1,38 & 12,1 & 120 & 178 \\
\hline & & & & & 16 & 1,57 & 12,4 & 51 & 91 \\
\hline & & & & & 18 & 2,46 & 13,3 & 51 & $12^{\prime}$ \\
\hline & & & & & 20 & 2,10 & 13,0 & 90 & 84 \\
\hline & & & & & 22 & 2,12 & 12,7 & 91 & 91 \\
\hline & & & & & 23 & 1,53 & 12,5 & 24 & 85 \\
\hline & & & & & 25 & 1,66 & 11,9 & 91 & 130 \\
\hline & & & & & 26 & 1.15 & 12,6 & 78 & 138 \\
\hline
\end{tabular}


L'étude statistique de l'intervalle de confiance de la population de ces catégories permet de classer un dosage quelconque de TGO dans l'un des trois groupes avec une erreur clinique n'excédant pas 5 p. Ioo.

$\mathrm{I}^{\mathrm{o}}$ Animaux sûrement sains (transminase inférieure à I30).

$2^{\circ}$ Animaux suspects (transaminase comprise entre 130 et 240 ).

$3^{\circ}$ Animaux sûrement malades (transminase supérieure à 240 ).

Les animaux étant ainsi groupés le tableau 2 fournit les taux de fer plasmatique dans ces différents groupes en fonction du taux de transminase libérée par la cellule.

Les groupes I et 2 sont pratiquement identiques ; par contre, ces groupes montrent avec le groupe 3 une différence hautement significative (test $F=6,98$, limite I p. IOO $=5,72$ ).

Enfin dans le tableau récapitulatif (tabl. 3) des résultats montrant les taux des différents éléments dans le plasma des veaux, on ne constate aucune anomalie, tant dans les taux de magnésium et de calcium que dans ceux de cuivre.

Ces éléments semblent donc à rejeter de l'étiologie du syndrome.

Les taux de magnésium chez les veaux sont normaux ou légèrement inférieurs à la normale, ce qui n'a rien de surprenant chez des animaux qui ne reçoivent que du lait; de toute façon on ne pourrait craindre un accident de tétanie par carence en magnésium qu'en dessous de $\mathrm{I} \mathrm{mg} / \mathrm{I}$ oo $\mathrm{ml}$ environ.

\section{DISCUSSION}

Les animaux n'ayant pas reçu la prophylaxie au sélénium se montrent cliniquement malades ou ont un taux de TGO élevé. Ces taux indiquent que les animaux sont atteints de myopathie à un stade subclinique (tabl. $\mathrm{x}$, colonne 2) et hormis les veaux $\mathrm{n}^{0} 7$ et $\mathrm{I} 8$, on peut penser $q \mathrm{u}^{\prime}$ une cause occasionnelle même minime serait susceptible de déclencher l'apparition du stade clinique.

Il est, en effet, classique de considérer l'effort violent et soudain, fou même le temps orageux) comme cause déclenchante de la maladie.

$L$ e tableau 2 nous indique que les animaux sains ou suspects ont des taux de fer plasmatique bas mais normaux pour des jeunes à la mamelle. Les animaux malades ayant une transminase élevée (supérieure à 240) ont des taux de fer élevés signifiant une lyse de la cellule et une mise en circulation massive de son fer.

Ce fait semblerait corroborer l'hypothèse de TERROINE (Ig64) selon laquelle une modification de la perméabilité cellulaire libérerait d'abord les enzymes, en phase aiguë de dystrophie musculaire.

En effet, l'augmentation du taux de fer dans le plasma n'est pas parallèle à l'augmentation du taux de transminase. Le fer n'est libéré dans le plasma que chez les malades graves et à taux de transminase supérieur à 240 .

La chronologie des événements semblerait donc être la suivante : de l'état normal la cellule subit une modification de perméabilité qui libère les enzymes, puis la lyse de la cellule elle-même libère les éléments constituants de cette cellule.

Annales de Biologie animale. - I 965 . 


\title{
CONCLUSION
}

Les animaux qui ont reçu une prophylaxie par le sélénium ont été efficacement protégés tandis que les animaux sains dans les exploitations atteintes de myopathie et ne pratiquant aucune prophylaxie étaient en fait, le plus souvent, des malades à un stade subclinique.

Les malades cliniques graves présentent une libération massive de fer dans le plasma.

I,e magnésium, le calcium et le cuivre, dans les conditions de cette étude, n'ont pas montré de variation dans cette maladie.

Rę̧u pour publication en janvier 1965 .

\section{REMERCIEMENTS}

Nous tenons à remercier le C. E. T. A. de Saint-Étienne-de-Fursac (Creuse) ainsi que les vétérinaires praticiens de cette région (Drs Chancel, LANSADE, SotTier, Dumas, PomareT) et de la région de Montluçon ( $\mathrm{D}^{r}$ PRAT) pour l'aide qu'ils nous ont apportée dans la réalisation de ce travail.

\section{SUMMARY}

\author{
MYOPATHY-DISPNOEA SYNDROME. INFLUENCE OF PREVENTIVE TREATMENT \\ WITH SELENIUM ON THE BIOCHEMICAL COMPOSITION OF PLASMA IN THE CALF
}

The biochemical composition of plasma was studied in 25 suckling calves from 15 establishments. In 8 of the establishments no preventive measures were taken. On the other 7 the dams were given $15 \circ \mathrm{g}$ daily of a mineral supplement containing $17.6 \mathrm{~g}$ sodium selenite per $100 \mathrm{~kg}$. From the first type of establishments there were 15 calves, 9 with clinical signs and 6 controls, and the Io calves from the second type were clinically healthy.

Glutamic oxaloacetic transaminase (GOT) in plasma was estimated by the method of REITMAN and FrANKEL. There was a highly significant difference between the healthy group which had been given preventive treatment and the two groups which had not been so treated ( $\mathrm{F}$ test $=20.4$;

I per cent limit $=5 \cdot 7^{2}$ ). On the other hand the difference between the healthy animals not treated and the sick animals was not significant.

The sick animals with GOT above 240 REITMAN-FRANKEI units had values for iron in plasma significantly higher than those with GOT below that value $(F$ test $=6.98$; I pour cent limit $=5.72)$.

The calves whose mothers had been given selenium were effectively protected. The controls which had not received preventive treatment were more often than not subclinical cases.

In the conditions of this trial magnesium, calcium and copper were nto changed by the disorder.

\section{RÉFÉRENCES BIBLIOGRAPHIQUES}

Blincoe C., Dye W. B., I958. Serum transaminase in white muscle disease. J. Anim. Sci., 17, 224-226. Cabaud P., LeEper R., Wroblewski F., I956. Colorimetric measurement of serum glutamic oxalacetic transaminase. Amer. J. Clin. Path., 26, i Ior-1 I03.

FaUvert R., HaRTMANN L., I962. Techniques modernes de laboratoire. I vol. 318 pages. L'Expansion scientifique. Éd. Paris. 
Henry R. J., Sobel C., Chiamori N., i958. Clinica Chim. Acta i958, 3, 523 (cités par Fauvert R., HARTMANN L., I962).

Jérome H., SchmitT H., I954. Microméthode spectrophotométrique de dosage du cuivre total dans les tissus et liquides biologiques. Bull. Soc. Chim. Biol., 9, 1343-1 354 .

McConnelt K. P., r963. Metabolism of selenium in the mammalian organism. Agric. Food. Chem. 11, 385-388.

KUTtLER K. L., MARBLE D. W., I958. Relationship of serum transaminase to naturally occurring and artificially induced white muscle disease in calves and lambs. Amer. J. Veter. Res., 19, 632-636.

LEwis W. H. P., I960. A micromethod for the estimation of magnesium. J. Med. Lab. Technol., 17, 32-55. Muth O H., Oldfield J. E., Remmert L. F., Scifubert J. R., 1958. Eiffects of selenium and vitamin E on white muscle disease. Science, 128, rogo.

Reitman S., Frankel S., I957. A colorimetric method for the determination of serum glutamic oxalacetic and glutamic pyruvic transaminases. Amer. J. Clin. Path., I957, 28, 56 (cité par FAUvERT R., et HARTMANN L., 1962.)

Sharman G. A. M., Blaxter K. L., Wilson R. S., I959. Prevention of enzootic Muscular dystrophy by selenium administration. Veter. Rec., $71,536$.

Terroine Th., r964. Le contrôle vitaminique des activités enzymatiques. Ann. Nutr. Alim., 18, 87-124, 